\title{
Evolutionary Learning Model of Social Networking Services with Diminishing Marginal Utility
}

\author{
Yutaro Miura \\ Department of Computer Science \\ and Communications Engineering, \\ Waseda University \\ y.miura@isl.cs.waseda.ac.jp
}

\author{
Fujio Toriumi \\ Graduate School of Engineering \\ The University of Tokyo \\ tori@sys.t.u-tokyo.ac.jp
}

\author{
Toshiharu Sugawara \\ Department of Computer Science \\ and Communications Engineering, \\ Waseda University \\ sugawara@waseda.jp
}

\begin{abstract}
We propose a model of a social networking service (SNS) with diminishing marginal utility in the framework of evolutionary computing and present our investigation on the effect of diminishing marginal utility on the dominant structure of strategies in all agents. SNSs such as Twitter and Facebook have been growing rapidly, but why they are prospering is unknown. SNSs have the characteristics of a public goods game because they are maintained by users posting many articles that incur some cost and because users can also be free riders, who just read articles. Thus, a number of studies aimed at understanding the conditions or mechanisms that keep social media thriving theoretically by introducing the meta-rewards game, which is a variation of a public goods game. The meta-rewards games assume constant marginal utility, meaning that the rewards by receiving comments increase linearly according to the number of comments, but describing the psychological rewards of humans is often inappropriate. In this paper, we present our modification of the model using the diminishing marginal utility and our comparison of the experimental results with those of the original meta-rewards game. We demonstrate that the structure of dominant strategies of all agents in our game is quite different from that in the original meta-rewards game and is more reasonable to explain the users' behavior in SNSs because their efforts in SNSs are limited even if they have many friends.
\end{abstract}

\section{CCS CONCEPTS}

- Human-centered computing $\rightarrow$ Social networks; • Computing methodologies $\rightarrow$ Multi-agent systems;

\section{KEYWORDS}

Social network systems; Cooperation; Prisoner's dilemma; Agent network; Evolutionary learning

\section{ACM Reference Format:}

Yutaro Miura, Fujio Toriumi, and Toshiharu Sugawara. 2018. Evolutionary Learning Model of Social Networking Services with Diminishing Marginal Utility. In WWW'18 Companion: The 2018 Web Conference Companion, April 23-27, 2018, Lyon, France. ACM, New York, NY, USA, 7 pages. https://doi.org/10.1145/3184558.3191573

This paper is published under the Creative Commons Attribution 4.0 Internationa (CC BY 4.0) license. Authors reserve their rights to disseminate the work on their personal and corporate Web sites with the appropriate attribution.

WWW'18 Companion, April 23-27, 2018, Lyon, France

(c) 2018 IW3C2 (International World Wide Web Conference Committee), published under Creative Commons CC BY 4.0 License.

ACM ISBN 978-1-4503-5640-4/18/04.

https://doi.org/10.1145/3184558.3191573

\section{INTRODUCTION}

Many social networking services (SNSs) on the Internet are growing and are widely used by close friend, acquaintances, and even complete strangers in communications such as opinion exchanges, publications of information, education [4], and advertising. Thus, understanding why people continue to submit content and respond to it is important. These activities are voluntary and incur certain costs; actually a few people may receive pragmatic rewards if their content in SNSs are aimed at improving sales revenue. However, a large amount of content is posted for psychological rewards as a kind of incentive. These people, for example, submit contents to receive positive reactions, which provide the feelings of approval, agreement, sympathy, etc., from their friends and acquaintances. Moreover, these interactions produce feelings of connections between people and a sense of belonging. However, it is still unknown whether or not such consecutive postings of articles and comments is really the dominant strategy for SNSs because some users can be free riders (or lurkers) who only read content; thus, they only receive a few rewards without any costs. Therefore, many studies focus on the mechanisms and design methodologies that lead to thriving SNSs using different approaches, such as social psychological studies [12], analyses of exchange patterns [6], evolutionary game theoretic approaches [16] and analyses of network structures and information diffusion $[11,14,15]$. We particularly focus on the evolutionary game theoretic method, which is a learning algorithm to understand how such incentives affect people's optimal strategy in SNSs.

A number of studies have been conducted according to this approach. For example, Toriumi et al. [16] and Hirahara et al. [10] discussed mechanisms to keep SNSs active by using an evolutionary game on a variety of network structures. They proposed a rewards game (RG) and meta-rewards game (MRG), which were dual parts of Axelrod's meta-norms game, to identify evolved behaviors of agents for a model of individual SNS users. They also analyzed the conditions for a cooperation dominant situation, which corresponds to when agents continue to post articles and comments. They then found that meta-rewards such as comments on article comments [16] and a simple (therefore low-cost) response mechanism for rewards such as "Like" buttons for articles [10] play an important role in SNSs. In addition, Osaka et al. [13] pointed out the possibility if direct reciprocity, which corresponds to the tendency that he commented on my article because I commented on his recent article and vice versa.

These studies assumed that the utility, which is the psychological rewards in these contexts, linearly increases as the number of comments on the posted articles increases. However, we believe 
that this assumption is untenable to describe personal utility in SNSs. Certainly, receiving a comment on one's own article is often happy and enjoyable. However, intuitively, the degree of happiness does not increase linearly but diminishes incrementally when so many comments are posted. For example, if we receive 100 positive comments, we do not feel 100 times of the happiness; this type of utility is often expressed by diminishing marginal utility. The model including diminishing marginal utility is also not always but is often used to characterize some sorts of risk averse attitude that is used to explain the relationships between the desired utility and cost (or risk) in the feeling of demand, such as with investment, income, and consumer goods.

Our research issue was to investigate how diminishing marginal utility in SNS communications affects users' strategy; in particular, we wondered if users with diminishing marginal utility would continue to post articles. For this purpose, we modeled users as agents connected with graphs, the links of which express the acquaintance relationships. To compare the effect of diminishing marginal utility and the constant marginal utility used in $[9,16]$, we investigated the difference in evolved strategies in agent network societies that have structures with a complete graph and ER networks. We show that a model with diminishing marginal utility evolves more realistic situations with the reasonable dominant strategy.

This paper is organized as follows. In Section 2, we describe a number of studies that attempt to understand the characteristics of social media and to identify why SNSs are prospering. Next, Section 3 explains the conventional rewards and meta-rewards games and introduces the meta-rewards game with diminishing marginal utility. Section 4 indicates that the meta-rewards game with diminishing marginal utility evolves quite a different structure of dominant strategies in agent networks, and their strategies are more acceptable and aptly describe human behaviors in SNSs. We also report the dynamical changes in the individual strategies over time. Finally, we conclude this paper with a summary and our future work.

\section{RELATED WORK}

Many studies have attempted to understand why people use social media platforms and social networking services by identifying what factors affect SNSs. Particularly, many empirical studies identify the reasons for continuous usage of social media (e.g. [1, 5]). For example, Al-Debei et al. [1] attempted to explain why people continuously use social media, specifically by focusing on Facebook, using the theory of planned behavior, which is a social psychological model to explain human intention and behavior. Chen et al. [5] analyzed the reasons for the continuous intention of use of social media from four social factors: subjective norms, images, critical mass, and electronic word-of-mouth. They revealed that user satisfaction with social media applications significantly affects electronic word-of-mouth, which in turn significantly influences their continuous intention of use. Lin and Lu [12] also empirically studied using social psychology the reasons people have for joining SNSs. They found that enjoyment is the most influential factor for people to continue using an SNS. They also found a notable difference between genders; i.e., the number of active peers is an influential factor for women's enjoyment, and it results in their continued use of social media, whereas the number of members does not have a significant effect on men's enjoyment.

Some researchers analyzed the characteristics of social media from the viewpoint of their network structures. For example, Karamon et al. [11] devised an algorithm that can analyze important network-based features of huge social networks for better understanding the user behavior therein. Saito and Matsuda [15] analyzed network structures to identify two key types of users who draw the attention of many other users on Twitter. They showed that one type has higher link reciprocity. Qasem et al. [14] attempted to detect influential actors in SNSs in order to identify who can influence others and to improve information diffusion and market efficiency in their networks.

Other studies proposed abstract models of social media and investigated their properties from a game theoretic approach. Anderson [2] proposed a game aimed at understanding how social media have emerged as a driving force in contemporary marketing and how this will affect future marketing. Xiong et al. [18] introduced the lurker game to model the dilemmas in human activities observed in big social networks. White et al. [17] tried to identify possible threats and the vulnerability to attack on online social media from game-theoretical and simulation-based viewpoints, and they analyzed several attack scenarios. Other game-theoretical studies on social network and social media can be found (e.g. [19]). To promote continuous usage of social networking services, Osaka et al. [13] extended the RG and MRG [16] to include reciprocity based on interaction patterns. They ran this game in a variety of complex networks and found that the behavior of the half-free rider, in which users comment on the articles posted by their close-friends but behave as free riders for other articles, is the best evolutionary strategy and maintains continuous use of SNSs. However, unlike our model in this paper, their studies assumed that the rewards/utilities used in fitness functions of an evolutionary game linearly increased according to the number of responses. We think that this assumption is not realistic because the rewards received by their followers's comments are psychological. Thus, we identified the effect of diminishing marginal utilities on the prosperity of and behaviors of users in SNSs.

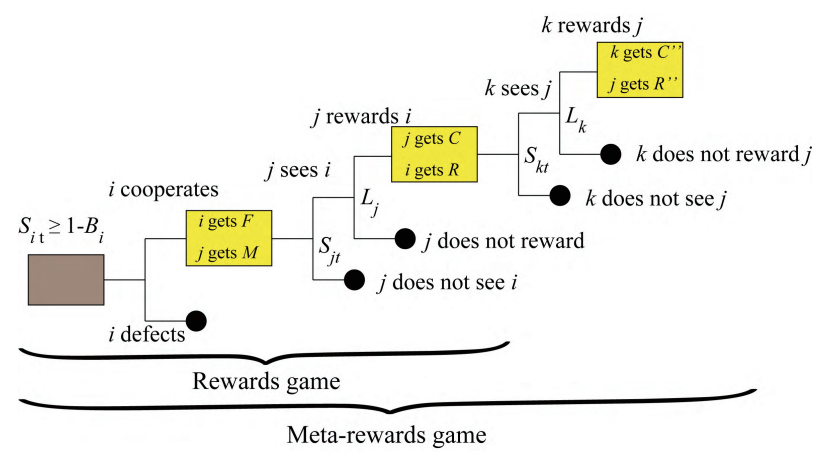

Figure 1: Reward and Meta-reward Games. 


\section{MODEL OF SOCIAL NETWORKING SERVICE WITH DIMINISHING MARGINAL UTILITIES}

\subsection{Reward and Meta-rewards Games}

SNSs are useful and can be active only if many users post articles and comment on the posted articles and if these articles and comments are shared with friends and/or anonymous participants. These activities incur some cost in terms of personal time and writing/reading efforts. Users can get some information through these activities and can also receive feelings of connectivity, empathy, and contentment by receiving reactions from other users. However, many free riders only obtain information by reading the content. Therefore, Toriumi et al. [16] pointed out that SNSs have the properties of public goods that are produced and maintained by cooperation in the SNS community. Such interaction structures of public goods have been studied for a long time, but the norms and meta-norms games proposed by Axelrod [3] are a notable work to define the game structure. Because SNSs have no mechanism of punishing non-cooperators, however, Toriumi et al. [16] defined a SNS as a dual part of the meta-norms game, which has a mechanism only for giving cooperators (psychological) rewards. Their framework includes two games, the rewards game (RG) and the meta-rewards game (MRG), corresponding to the norms game and meta-norms game, respectively. They indicated that meta rewards facilitate cooperation, thereby resulting in active use of an SNS; this result is similar to the results in a dual space by Axelrod [3], who found that punishment to non-cooperators is the key mechanism to maintaining shared goods. Note that the game structure of a RG and MRG is, like a meta-norms game, essentially an $n$-person prisoner's dilemma (PD) game in which cooperation is "posting articles and comments" and defection is "just reading while posting no articles and comments."

Let $A=\{1, \ldots, n\}$ be the set of $n$ agents that interact with other agents according to a network structure, which is represented by graph $G=(A, E)$, where $E$ is the set of edges that correspond to friend/acquaintance relationships. For $\forall i \in A$, the set of neighbor agents of $i$ is denoted by $N_{i} \subset A$. Agents have two possible actions, cooperation (posting articles and comments) or defection (just reading them without posting any information). Agent $i \in A$ has two learning parameters to specify the probability of cooperation, $B_{i}$ (posting a new article) and $L_{i}$ (posting a comment on the article and a comment on comments), as shown in Fig. 1. The meanings of other parameters that affect agent's activities in Fig. 1 are listed in Table 1. The parameters in this table were determined based on the experiments by Axelrod [3] and were common among all agents.

MG and MRG proceed as follows (also see Fig. 1). When agent $i \in A$ attempts to post an article at game round $t$, a number, $S_{i}^{t}$, is randomly selected from interval $[0,1]$. Intuitively, $S_{i}^{t}$ expresses the degree of fun of (or the degree of attention to) the content of the article that $i$ is going to post and the associated comments after that. Therefore, $i$ decides to post an article with $\operatorname{cost} F(<0)$; otherwise $\left(S_{i}^{t}<1-B_{i}\right), i$ does not post it (i.e., defeat), and the game flow ends. When $i$ posts it, its friend agent $\forall j \in N_{i}$ that gains reward $M$
Table 1: Parameters in rewards and meta-rewards games.

\begin{tabular}{lc}
\hline \multicolumn{1}{c}{ Meaning } & Parameters \\
\hline Cost of article post & $F$ \\
Reward from reading an article & $M$ \\
Cost of comment & $C$ \\
Reward from reading a comment & $R$ \\
Cost of comment return & $C^{\prime \prime}$ \\
Reward from comment returns & $R^{\prime \prime}$ \\
\hline
\end{tabular}

$(>0)$ from the posted article with probability $S_{i}^{t}$ by reading $i$ 's article. The games for the neighbor agents that do not read it end here. Another agent $j$ posts a comment on the article with probability $L_{j}$ and pays $\operatorname{cost} C(<0)$. Then, $i$ gains reward $R(>0)$ through each of the comments by $i$ 's neighbors. The game flow so far is a rewards game.

In a meta-rewards game, the meta-rewards process is added to the rewards game: After $j$ 's comment on $i$ 's article, agent $k \in N_{j}$ finds and reads $j$ 's comment and decides to post a comment on it with probability $L_{k}$. If $k$ posts it, $k$ pays cost $C^{\prime \prime}(<0)$, and $j$ gains reward $R^{\prime \prime}(>0)$. We assume that, in a (game) round, only one agent has a chance to post an article, may receive comments, and reply to some of these comments. The value of $S_{i}^{t}$ is randomly selected to determine whether or not agents post an article. It is also used in deciding whether or not agents read the posted articles and comments [3]. Thus, if $S_{i}^{t}$ is low, other agents have difficulty in to reading/noticing the posted article and its comments, and only agents having relatively high $B_{i}$ post an article.

\subsection{Diminishing Marginal Utility}

However, RG and MRG assume that the psychological utility is linearly correlated with the number of comments, and we believe that it is counter-intuitive to our actual feelings of utility. If we consider the Weber-Fechner law [7, 8], marginal utility decreases according to the number of comments, like the utility value observed in income based on monetary value. Thus, we integrated the concept of diminishing marginal utility into RG and MRG. The difference between linear and diminishing marginal utilities are illustrated in Fig. 2. We assume that marginal utility increases logarithmically according to the number of comments on an article and the number of comments on a comment [8]. However, we assume the cost linearly increases according to the number of posts and comments.

Therefore, we define the utility of receiving comments after $i$ 's posting article, $a$, as

$$
R \times \log _{e}\left(N_{c}(a)+1\right),
$$

where $N_{c}(a)$ is the number of comments on $a$ posted by $i$ 's neighboring agents. For example, if $i$ posts article $a$, receives $N_{c}(a)$ comments, and posts $N_{c c}(a)$ comments on the received comments, then $i$ 's utility value in this round of our game will be

$$
F+R \times \log _{e}\left(N_{c}(a)+1\right)+C^{\prime \prime} \times N_{c c}(a) .
$$

Note that $F$ and $C^{\prime \prime}$ are negative values. However, agent $j \in N_{i}$ that reads $a$ and posts comment $c$ on article $a$ gains $M$ and pays cost $C$, and if $j$ receives $N_{m}(c)$ comments on $c$, it gains $R$; thus, the 
total utility value is

$$
M+C+R^{\prime \prime} \times \log _{e}\left(N_{m}(c)+1\right) .
$$

The RG and MRG with diminishing marginal utility are denoted by $d R G$ and $d M R G$, after this. Note that in Toriumi et al. [16], the utility value of the comments on the posting article $a$ is defined as $R \times$ $N_{c}(a)$; thus, obviously the utility value in $\mathrm{d}(\mathrm{M}) \mathrm{RG}$ is considerably lower than that in $(\mathrm{M}) \mathrm{RG}$.

\subsection{Evolution in rewards and meta-rewards games}

The dRG and $\mathrm{dMRG}$ are formulated in the framework of the evolutionary game - as they are in the meta-norms game - in order to find the best strategy in these games for individual agents in the population of agents connected with the given network structure. The learning parameters $B_{i}$ and $L_{i}$ for $\forall i \in A$ are represented in a 3-bit gene, so $i$ has a 6-bit gene. This encoding is based on that in Axelrod [3] and identical to that in Toriumi et al. [16]. The initial values of $B_{i}$ and $L_{i}$ are randomly defined.

One generation of games is defined as a term in which each agent has four chances to post articles, thereby consisting of $4 \times$ $|A|$ rounds. Then, the agent selects two agents as parents from itself and its neighboring agents using the roulette-wheel selection based on the fitness values in their environments (selection), and it generates a child agent for the same node of the network in the next generation (crossover). The fitness value for agent $i$ is defined as the sum of the utility value received minus costs incurred during the current generation. We assume the diminishing marginal utility as defined in Formula (1). Then, each bit of the selected gene is inverted with some probability (mutation). This probability was set to 0.005 in the following experiments. We continued this up to 10,000 generations. Note that parameters $B_{i}$ and $L_{i}$ are expressed by a three-bits gene, so they take on a discrete value, i.e., $0 / 7,1 / 7$, $\ldots$, or $7 / 7$.

\section{EXPERIMENTAL RESULTS}

The purpose of our experiments was for agents to learn the best behavioral strategy in the game theoretic model of SNSs with diminishing marginal utility, and we compared the results with those when the original MRG had its utility linearly increase as the number of received comments increased. We also investigated whether their strategies dynamically changed or remained fixed over 10,000

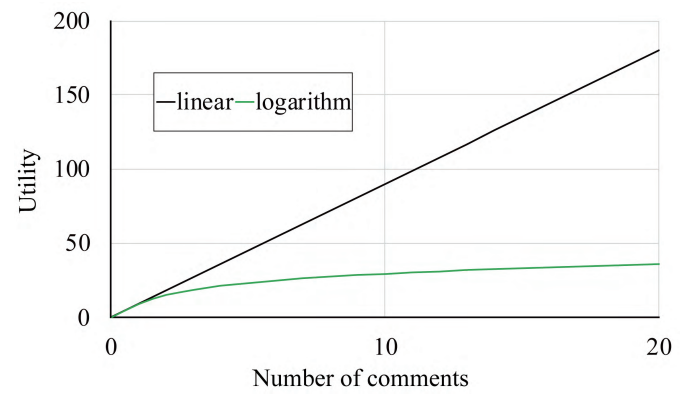

Figure 2: Difference in increase of utility when $R=9$.
Table 2: Initial values of variables and learning parameters.

\begin{tabular}{cll}
\hline Parameter & Description & Value \\
\hline$A$ & Set of agents & $|A|=20$, 50, or 1000 \\
$B_{i}$ & $\begin{array}{l}i \text { 's probability of posting } \\
\text { an article }\end{array}$ & random (3-bit gene) \\
$L_{i}$ & $\begin{array}{l}i \text { 's probability of posting a } \\
\text { comment }\end{array}$ & random (3-bit gene) \\
$S_{i}^{t}$ & Degree of fun of $i$ ' article & $0 \leq S_{i}^{t} \leq 1$ (ran- \\
$F$ & Cost of posting article & -3.0 \\
$M$ & $\begin{array}{l}\text { Reward for reading article } \\
\text { Costs of comment and }\end{array}$ & -2.0 \\
$C$ and $C^{\prime \prime}$ & $\begin{array}{l}\text { Comment return } \\
\text { comm and } R^{\prime \prime}\end{array}$ & $\begin{array}{l}\text { Rewards for receiving } \\
\text { comment and comment }\end{array}$ \\
& return \\
\hline
\end{tabular}

generations and how the characteristics of the network structure affected on their strategy. The initial values of the game and learning parameters are shown in Table 2. The results shown below are the average values of independent 100 experimental runs based on different random seeds, unless otherwise noted. Note that the dominant strategy in RG and dRG was defection, like the norms game, and we omit showing the results of RG and dRG.

\subsection{Effect of diminishing marginal utility on cooperation}

In the first experiments, we compared the evolved strategies, which are a collection of the best strategies for an individual agent, in dMRG with those in MRG. In these experiments, we assumed that the network is a complete graph, the number of nodes of which is twenty $(|A|=20)$, because the results of this network exhibited the basic characteristics of dMRG and MRG, and Toriumi et al. [16] also conducted their experiments using the complete graph. The results of agents' activities are plotted in Fig. 3, where the average probabilities of article post, $B$, and comment post, $L$, in MRG and

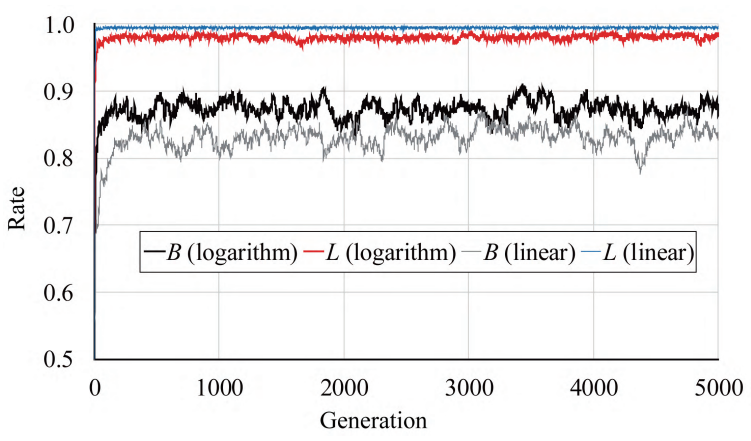

Figure 3: Posting $(B)$ and comment $(L)$ rates in dMRG and MRG in complete graphs. 


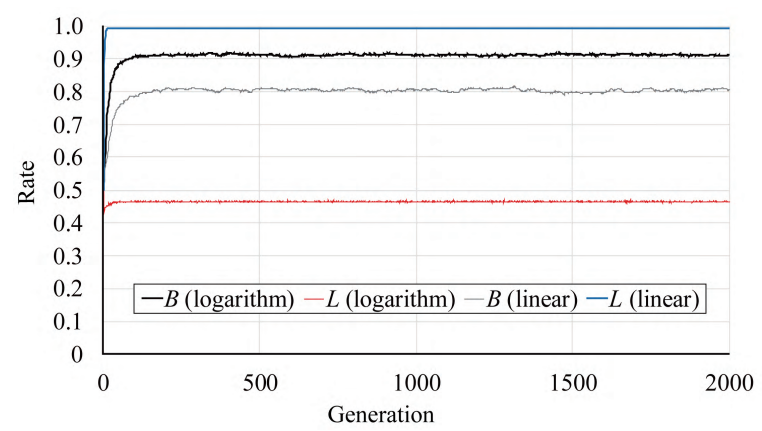

Figure 4: Posting $(B)$ and comment $(L)$ rates in dMRG and MRG in ER networks.

dMRG are compared. Note that $B$ and $L$ are calculated as

$$
B=\sum_{i \in A} B_{i} /|A| \text { and } L=\sum_{i \in A} L_{i} /|A| .
$$

These are called the posting rate and comment rate after this. The results of MRG and dMRG are shown with the labels of linear and logarithm, respectively, in this and the subsequent figures.

Figure 3 indicates that posting rate, $B$, in MRG (gray line) fluctuated approximately between 0.80 and 0.85 (the average is around 0.830 ), and the comment rate, $L$, in MRG (blue thin line) almost constantly maintained 1.0 ; these results are consistent with those of Toriumi et al. [16]. However, $B$ in dMRG (black line) fluctuated approximately between 0.85 to 0.90 (the average was 0.872 ), and $L$ in $\mathrm{dMRG}$ (red line) was around 0.98 , slightly lower than $L$ in MRG. These results indicate that the evolved dominant strategy in dMRG was cooperation, and thereby, agents continued to post articles. A comparison of the results of $\mathrm{dMRG}$ with those of MRG shows that the cooperation in $\mathrm{dMRG}$ can be maintained with a slightly lower comment rate than that in MRG, although the posting rate was higher in dMRG.

We also conducted the same experiment using the Erdős-Rényi random network (ER network), where the number of nodes was 1000 and where the average degree was 50 . The results of the evolved strategy are plotted in Fig. 4. This figure clearly shows that the cooperation was maintained ( $B$ was around 0.91 ) with a much lower comment rate ( $L$ was around 0.46 ) in $\mathrm{dMRG}$, but the posting and comment rates in MRG was similar to the results in the complete graph in Fig. 3 (although $B$ fluctuated more in Fig. 3).

\subsection{Degree and Comment Rate}

Figures. 3 and 4 also show that the comment rates in these networks were quite different in $\mathrm{dMRG}$, although they were not so different in MRG. To see what network characteristic caused this difference, we investigate how posting and comment rates changed depending on the average degree in ER networks. The experimental results are shown in Fig. 5. In MRG, the comment rate, $L$, remained unchanged at approximately 1.0, and the posting rate $B$ also remained at around 0.8 , though it slightly decreased as the (average) degree of the ER network increased. In dMRG, the comment rate decreased as the degree increased, but $B$ remained around 0.9.

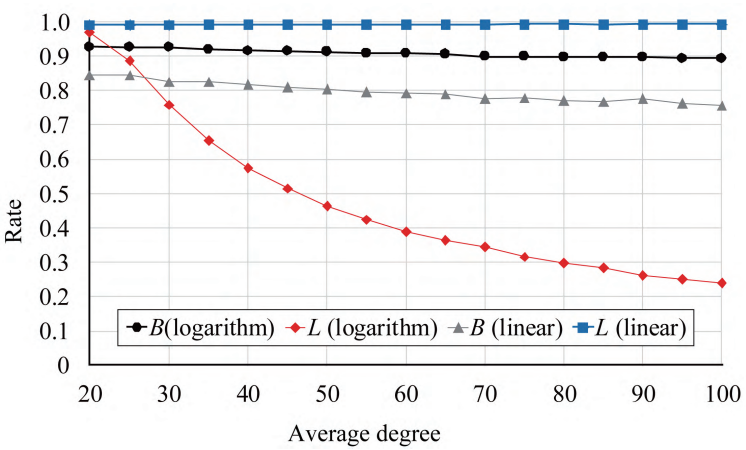

Figure 5: Posting and comment rates in ER networks with various degrees.

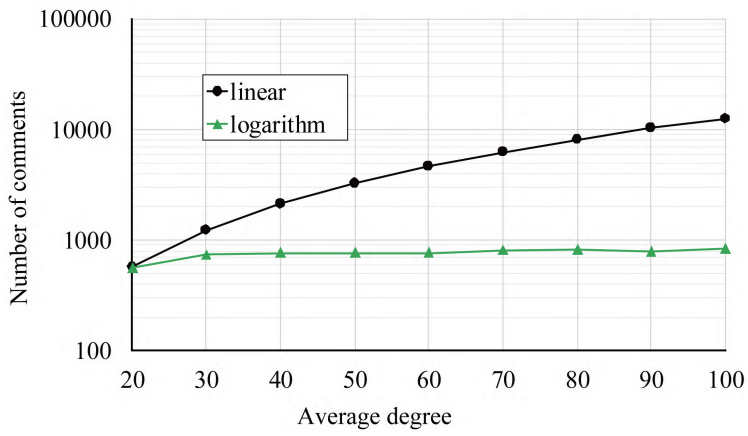

Figure 6: Number of comments for 5000 generations per agent in ER networks.

We can also see that the posting rate in $\mathrm{AMRG}$ was always higher than that in MRG.

These results mean that $B$ was maintained high (i.e., SNS prospered) with many fewer comments (i.e., fewer rewards and lower efforts) in dMRG. Moreover, this feature should not be overlooked: agents who contributed to the SNS by posting articles could gain rewards from the comments from their neighbors but could continue to post with fewer rewards in dMRG. Conversely, agents who commented on articles could reduce their efforts (or costs). Because $L$ was near 1.0 in the original MRG, agents had to comment on almost all articles, which obviously increased as the degree (the number of neighbors) increased: This result deviated from actual human behavior because the efforts were always limited. However, in the dMRG, the comment rates decreased with the increase of the average degree. Figure 6, which plots the average number of comments in 5000 generations per agent, shows that the number of comments increased exponentially in MRG (the vertical axis of Fig. 6 is a logarithmic scale), but the number in dMRG was almost constant. Because we cannot provide infinite efforts to SNSs, these results indicate that the $\mathrm{dMRG}$ is more appropriate to model the actual behaviors of humans in SNSs. Note that we also conducted the same experiments using a complete graph; their results were almost identical to those shown in Figs. 5 and 6, although the number of nodes increased in accordance with the number of the average degree. Thus, the decrease of $L$ in dMRG was clearly 


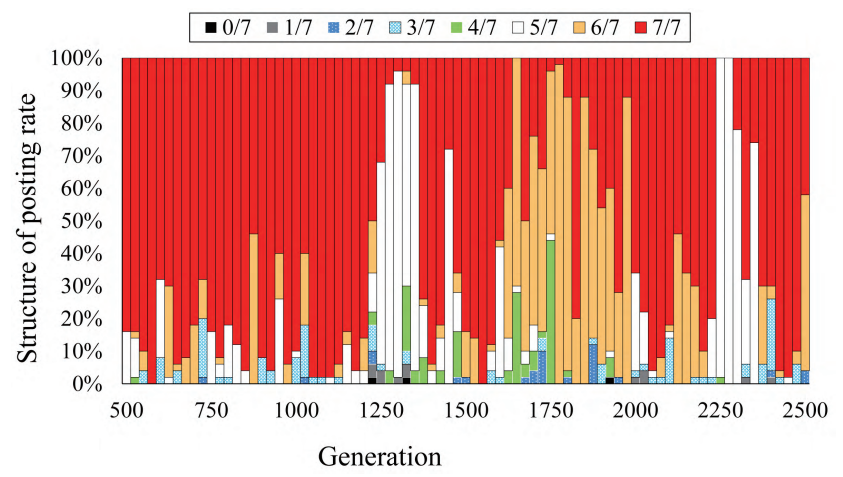

(a) Structure of posting rates for all agents

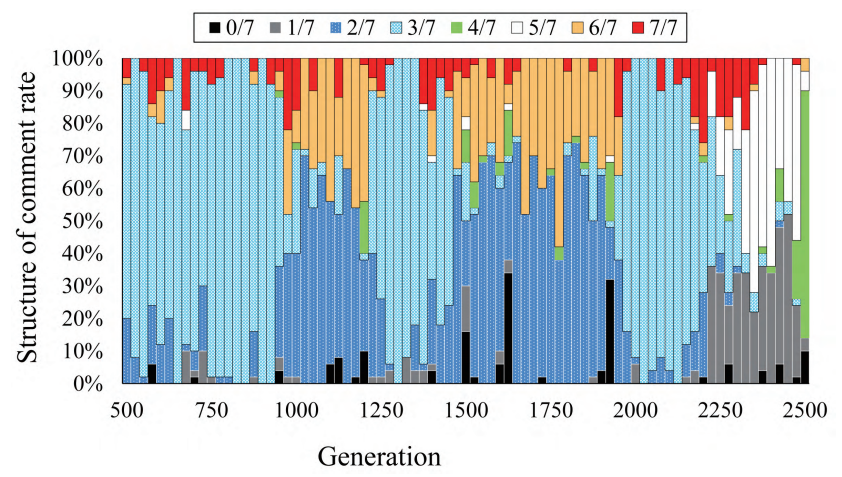

(b) Structure of comment rates for all agents

Figure 7: Structure of strategies of agents in dMRG (complete graph).

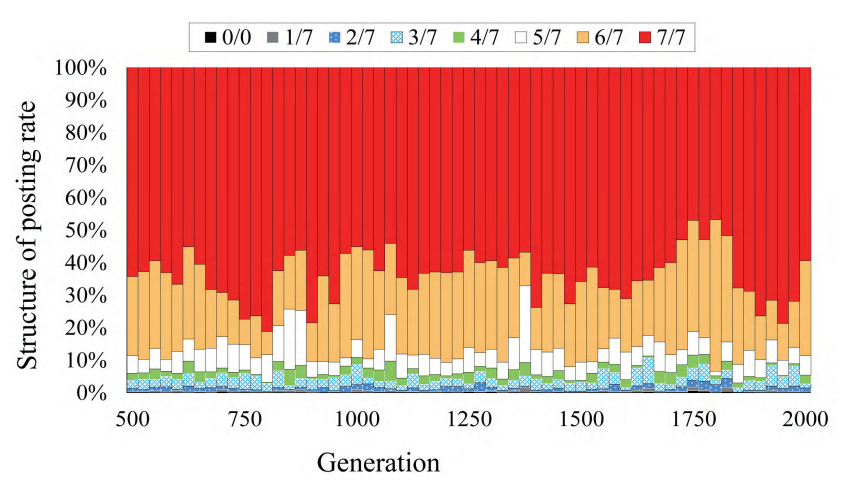

(a) Structure of posting rates for all agents

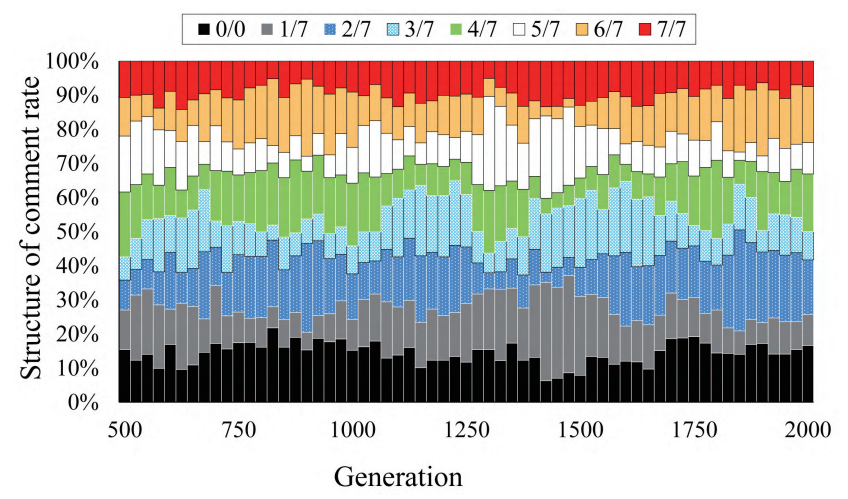

(b) Structure of comment rates for all agents

Figure 8: Structure of strategies of agents in dMRG (an ER network).

affected by the number of neighbor (acquaintance/friend) agents, not by the agent population.

\subsection{Structure of strategies over generations}

We conducted an experiment to investigate the structure of the emerging strategies in agents. We illustrated the distribution of the values of $B_{i}$ and $L_{i}$ between 500 and 2500 generations using an instance of an experimental run in Fig. 7 (complete graph) and Fig. 8 (an ER network). We set the number of agents to fifty $(|A|=50)$ so that their average degrees of nodes were almost identical. Note that this instance was randomly selected and that others also exhibited a similar phenomenon. These graphs reveal quite different characteristics in their structure of the selected strategies. In the complete graph, the structure varied frequently; for example, in Fig. 7(a), $B_{i}=7 / 7$ was the majority until 1200 generations, but the majority suddenly lowered to $5 / 7$ until around 1350 . After that, the agents recovered to $B_{i}=7 / 7$, and this kind of fluctuation also iterated after 2500 generations. Similarly, from Fig. 7(b), we could observe three main structures in the value of $L_{i}$ : the majority of agents selected (1) $L_{i}=3 / 7$, (2) $L_{i}=6 / 7$ and 2/7, and (3) $L_{i}=5 / 7$ and $1 / 7$; these structures changed dynamically over generations. However, their structure continued to be come more stable over generations in an ER network (Fig. 8) than that in a complete graph. Note that Fig. 8 (b) indicates that approximately $10 \%$ of agents have $L_{i}=0$, but of course the values of $L_{i}$ in individual agents varied.

We believe that this difference is caused by the density of clusters (or the cluster coefficient). In the complete graph, which is the instance of an extremely dense network, agents selected the genes on the basis of the utilities earned among all agents with a certain ratio. However, due to the mutation, a few genes changed. Even the $L_{i}$ in a certain agent suddenly became high, and the utility values around $i$ did not increase very much due to diminishing marginal utility. Another agent could make $L_{j}$ lower (because lower $L_{j}$ was beneficial for $j$ in this situation). Because all agents were fully connected, this change affected agents globally, thereby causing the changes in all of them. On the other hand, in ER networks, such changes might have affected only the neighbor agents, and they were absorbed locally, resulting in the more stable structure of the ratios of strategies.

\subsection{Discussion}

The experimental results enable us to say that the structure of the dominant strategies evolved in dMRG is more reasonable and reflects more acceptable behaviors than that in the original MRG. 
Because dMRG reduces the utility, we first thought that the posting rate would decrease, but the results were counter-intuitive. In the MRG, the utility increases linearly according to the number of comments, so receiving more comments increases the value of the fitness function in all agents. However, this strategy also raises the cost, which increases linearly according to the number of posted comments, so the posting rate remains slightly lower than that in dMRG. In the dMRG, however, receiving so many comments results in limited happiness, but posting comments on all articles linearly increases the cost of efforts. Thus, a certain level of comment rating leads to the best strategy. In such an equilibrium situation, the posting rate is maintained slightly higher with a very low comment rate. This limits the number of comments, making this situation reasonable in the model of actual human behavior.

Our experiment also suggested that the structure of strategy may be unstable in a dense cluster. Because actual social networks consist of sense and coarse clusters; a number of dense clusters are loosely connected. Thus, we can expect that activities in a cluster dynamically change, even though the relationships between clusters are more stable. However, we need to conduct more experiments to clarify it.

\section{CONCLUSION}

We proposed a model of SNS called a meta-rewards game with diminishing marginal utility and compared the evolved structure of dominant strategies in agent networks with that in a game with constant marginal utility, where the evolved dominant strategies correspond to the best behavioral strategies for individual agents. Our experiments showed that cooperation (posting articles) emerged with a lower value of comment rate in dMRG than that in MRG. Our experiments also revealed that the evolved structure of the dominant strategy became unstable in the dense networks. We plan to conduct more experiments to clarify the stability/instability in individual behaviors over generations.

\section{REFERENCES}

[1] Mutaz M. Al-Debei, Enas Al-Lozi, and Anastasia Papazafeiropoulou. 2013. Why people keep coming back to Facebook: Explaining and predicting continuance participation from an extended theory of planned behaviour perspective. Decision Support Systems 55, 1 (2013), 43 - 54. https://doi.org/10.1016/j.dss.2012.12.032

[2] Eric Anderson. 2010. Social Media Marketing - Game Theory and the Emergence of Collaboration. Springer, Berlin, Heidelberg.

[3] Robert Axelrod. 1986. An Evolutionary Approach to Norms. The American Political Science Review 80, 4 (1986), 1095-1111. http://www.jstor.org/stable/1960858
[4] Antoni Calvó-Armengol, Eleonora Patacchini, and Yves Zenou. 2009. Peer Effects and Social Networks in Education. The Review of Economic Studies 76, 4 (2009), 1239-1267. https://doi.org/10.1111/j.1467-937X.2009.00550.x

[5] Shih-Chih Chen, David C. Yen, and Mark I. Hwang. 2012. Factors influencing the continuance intention to the usage of Web 2.0: An empirical study. Computers in Human Behavior 28, 3 (2012), 933 - 941. https://doi.org/10.1016/j.chb.2011.12.014

[6] Samer Faraj and Steven L. Johnson. 2011. Network Exchange Patterns in Online Communities. Organization Science 22, 6 (2011), 1464-1480. https://doi.org/10.1287/orsc. 1100.0600

[7] Gustav Fechner. 1966. Elements of psychophysics. Vol. I. New York.

[8] Hermann Heinrich Gossen. 1983. The laws of human relations and the rules of human action derived therefrom. Mit Press.

[9] Yuki Hirahara, Fujio Toriumi, and Toshiharu Sugawara. 2013. Evolution of Cooperation in Meta-rewards Games on Networks of WS and BA Models. In Proc. of the IEEE/WIC/ACM Int. Joint Conf. on Web Intelligence (WI) and Intelligent Agent Technologies (IAT), Vol. 3. IEEE, 126-130.

[10] Yuki Hirahara, Fujio Toriumi, and Toshiharu Sugawara. 2016. Cooperationdominant Situations in SNS-norms Game on Complex and Facebook Networks. New Generation Computing 34, 3 (2016), 273-290. https://doi.org/10.1007/s00354-016-0305-z

[11] Jun Karamon, Yutaka Matsuo, and Mitsuru Ishizuka. 2008. Generating Useful Network-based Features for Analyzing Social Networks. In Proceedings of AAAI 2008. 1162-1168.

[12] Kuan-Yu Lin and Hsi-Peng Lu. 2011. Why people use social networking sites: An empirical study integrating network externalities and motivation theory. Computers in Human Behavior 27, 3 (2011), 1152 - 1161. https://doi.org/10.1016/j.chb.2010.12.009 Group Awareness in CSCL Environments.

[13] Kengo Osaka, Fujio Toriumi, and Toshihauru Sugawara. 2017. Effect of direct reciprocity and network structure on continuing prosperity of social networking services. Computational Social Networks 4, 1 (2017), 2. https://doi.org/10.1186/s40649-017-0038-2

[14] Ziyaad Qasem, Marc Jansen, Tobias Hecking, and H. Ulrich Hoppe. 2017. Influential Actors Detection Using Attractiveness Model in Social Media Networks. In Complex Networks \& Their Applications V: Proceedings of the 5th International Workshop on Complex Networks and their Applications (COMPLEX NETWORKS 2016), Hocine Cherifi, Sabrina Gaito, Walter Quattrociocchi, and Alessandra Sala (Eds.). Springer International Publishing, Cham, 123-134. https://doi.org/10.1007/978-3-319-50901-3_10

[15] Kodai Saito and Naoki Masuda. 2013. Two types of Twitter users with equally many followers. In Proc. of the 2013 IEEE/ACM Int. Conf. on Advances in Social Networks Analysis and Mining. ACM, 1425-1426.

[16] F. Toriumi, H. Yamamoto, and I. Okada. 2012. Why Do People Use Social Media? Agent-Based Simulation and Population Dynamics Analysis of the Evolution of Cooperation in Social Media. In 2012 IEEE/WIC/ACM International Conferences on Web Intelligence and Intelligent Agent Technology (WI-IAT), Vol. 2. 43-50. https://doi.org/10.1109/WI-IAT.2012.191

[17] J. White, J. S. Park, C. A. Kamhoua, and K. A. Kwiat. 2013. Game theoretic attack analysis in Online Social Network (OSN) services. In 2013 IEEE/ACM International Conference on Advances in Social Networks Analysis and Mining (ASONAM 2013). 1012-1019. https://doi.org/10.1145/2492517.2500257

[18] X. Xiong, D. Jiang, Y. Wu, L. He, H. Song, and Z. Lv. 2017. Empirical Analysis and Modeling of the Activity Dilemmas in Big Social Networks. IEEE Access 5 (Nov. 2017), 967-974. https://doi.org/10.1109/ACCESS.2016.2626079

[19] Narahari Yadati and Ramasuri Narayanam. 2011. Game Theoretic Models for Social Network Analysis. In Proceedings of the 20th International Conference Companion on World Wide Web (WWW'11). ACM, New York, NY, USA, 291-292. https://doi.org/10.1145/1963192.1963316 